\title{
Comparative Study on Mobile Wireless Sensor Network Testbeds
}

\author{
Osman Khalid and Muhammad Sualeh
}

\begin{abstract}
Anexperimental platform that provides an opportunity for researchers to physically test the real-time behavior of mobile wireless sensor nodes is called a Testbed. A lot of work is already done in the testbeds of Wireless Sensor Networks (WSN) with static nodes. However, there are a few testbeds that provide testing for sensor nodes with pure mobility. This paper performs a comparative studyonly for Mobile Wireless Sensor Network Testbeds (MWSNTs) with an intention to provide a quick reference for the current trends in the research conducted on testbeds. The paper discusses the various parameters that have to be taken into consideration, such as: mobility, control, medium access, energy utilization, localization, hardware/software requirements, and outlines the major challenges in the designing of MWSNTs. This paper infers that MWSNTs provide an interesting area of research in the designing and deployment of autonomous mobile wireless sensor devices.
\end{abstract}

Index Terms-Mobility, simulator, testbeds, mobile wireless sensor network testbeds (MWSNTs).

\section{INTRODUCTION}

A number of software simulators have beendeveloped to mimic the physical environment of mobile wireless sensors in software based environment. However, various experiments have indicated that there are significant differences in the results produced under the theoretical or simulated environment as compared to the results produced through empirical ways. These differences in results are due to the real-time radio signal propagation effects such as reflection, diffraction, multipath, free space loss and noise. Therefore, efforts are done to overcome software based experimentation limitations by designing the physical testbeds for mobile WSNs. In some efforts the testbeds with static nodeswere used to mimic mobilityby radio signal attenuation but still could not producerealistic results.

Among major challenges in designing of mobile WSN testbeds is to handle the mobility, maintenance, and interfacing of devices. The mobility can be provided by mounting the sensor nodes on small sized robots. Here, mobility pattern and node localization is also needed to be defined that the robots will follow. The different mobility patterns can also be controlled by using Player/Stage API [1].The maintenance of mobile nodes is also a major problem.In order to provide a $24 \times 7$ operation of the testbed, the mobile nodes should have auto-recharging capability. Firstly, for indoor testbeds, an uninterrupted operation can be maintained by fixing some docking stations monitoring the

Manuscript received September 15, 2012; revised December 7, 2012.

Osman Khalid is with Comsats Institute of Information Technology, Pakistan (e-mail: osmankhalid2005@gmail.com)

Muhammad Sualeh is with Chalmers University, Sweden. battery power of nodes and nodes automatically move to the docking station if the power drops below a certain threshold. Secondly, for outdoor testbeds, the solar panels can be used to auto-recharge the batteries. Thirdly, for localization the centralized or distributed mechanisms can be employed and finally, an interface is required so that the user can perform the experiment using testbed interface. The existing interfaces of most of the MWSNTs are the 'on the site interfaces' which means the interfaces are located at the testbed site and cannot be accessed remotely. However, most of the static WSN testbeds provideremote, online interface, such as Quri Nettestbed [2].

Several initiatives are already taken to address the above mentioned challenges, in the development of various testbeds. A few ofsuch testbeds are included in this paper in order to give an idea about the kind of workalready done and what are the future trends in research. The rest of the paper is organized as follows. In Section II, a brief study of testbeds for different selected parameters (such as infrastructure, deployment, mobility, auto-recharging, localization, collision, cost, and user interface) is presented. In Section III, a quantitative and qualitative comparison of selected testbeds is shown in tabular form. Section IV concludes the paper, highlighting current trends and a few suggestions for future work in development of MWSNTs.

\section{EXISTINGMWSN TESTBEDS}

Following are the various mobile WSN testbeds developed till now along with their infrastructure details and related parameters.

\section{A. MiNT-M: An Autonomous Mobile Wireless Experimentation Platform}

Miniaturized Network Testbed for Mobile Wireless Research (Mint-m) is a project initiated by researchers of Department of Computer Science of Stony Brook University, Stony Brook, New York [3].It is an indoor testbed.

\section{1) Major components}

Mint-m uses a wireless device called Router BOARD 230 which is mounted on the Roomba robot. The Router BOARD has four wireless interfaces each provided by a mini-PCI IEEE $802.11 \mathrm{a} / \mathrm{b} / \mathrm{g}$ wireless card. The four cards allow the nodes to be used in multi-radio experiments. In order to keep the testbed area smaller, the radio signal attenuators are used between a wireless interface and its antenna to decrease the signal powers and ultimately decreasing the physical space requirement. The major components are shown in Fig. 1.

2) Mobility

For mobility, MiNT-m uses a low cost robotic vacuum cleaner called Roomba. Roomba is an externally controlled 
and self-operating robotic vacuum cleaner developed by iRobot, and also has the auto recharging capabilities [4]. It can carry a weight of up to 30 pounds and one robot costs $\$ 250$.

\section{3) Auto-recharging}

MiNT-m testbed supports auto-recharging of mobile devices. Roomba robot comes with auto-recharging features; however, the circuitry is modified to provide power to the wireless router mounted on the robot. The devices are provided with a self-docking and charging mechanism. When the power of the mobile device drops below a certain threshold, it searches for a nearby docking station that is emitting an infrared beacon to indicate its position. The device then homes itself on the docking station to get auto-recharge.

\section{4) Localization}

The testbed uses commercial off the shelf available webcams to monitor the position of nodes with accuracy. The webcams are installed with the ceiling of the indoor site. Each wireless device has a board attached having a different color combination. The vision-based positioning system uses these color combinations in estimating and planning the trajectories of the robots to make a collision free movement of nodes. The Roomba' smovement is controlled by Spitfire Universal Remote Controller.

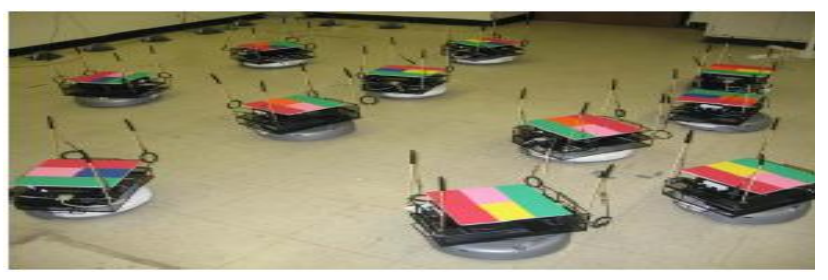

Fig. 1. Roomba robots with mounted wireless routers and the docking stations on top left corner [4].

\section{B. Mobile Emulab: A Robotic Wireless and Sensor Network Testbed}

Mobile Emulab is a project initiated by University of Utah, School of Computing and Department of Mechanical Engineering [5]. The testbed is indoor, deployed at L-shaped area with 6 mobile robots, and a number of fixed motes are attached on the sides of the mobility area.

\section{1) Major components}

Each robot consists of wireless $802.11 \mathrm{~b}$ card, the onboard computer, and a Mica2 mote. The wireless card provides the wireless communication of robot with the main testbed and the Internet. The onboard computer is a small computer running Linux operating system providing control to the user to run any code and the communication with the mote. The robot runs on battery with 2 to 3 hours life, but with no auto-recharging facility. Each robot is also mounted with a color pattern that is recognized by the cameras fitted in the ceiling, to calculate the position and next movement of the robot.

\section{2) Mobility}

For providing mobility to wireless sensor devices, Emulab uses Acroname Garcia robots which are two wheeled, small sized, and having options for custom configurations that buyers determine [6].

\section{3) Localization}

For localization and identification, two main components are responsible in Emulabtestbed: (a) visiond and (b) robotd.
The visiond uses ceiling-mounted low cost wide angle cameras to locate and track the robots. Each camera is aimed at a specific mark on the ground to keep the proper direction and alignment. The researcher first scripts the robot movements in NS file, and then robotd gives directions to the robot to reach the scripted destination points, and also avoids the robot collisions.

\section{Pharos: An Application-Oriented Testbed for}

Heterogeneous Wireless Networking Environments

The Pharos project has been initiated by the research group of The University of Texas at Austin, USA. This is an out-door testbed with robots being used to provide mobility [7].

\section{1) Major components}

The basic components include x86 Linux motherboard attached with Freescale microcontroller. This hardware supports a variety of devices that can be plugged in, like a range of $\mathrm{I} / \mathrm{O}$ devices and different sensors. For wireless communication an IEEE $802.11 \mathrm{~b} / \mathrm{g}$ wireless card is used with $5.5 \mathrm{~dB}$ antenna. The mobile node Proteus also supports other technologies, such as Bluetooth and Crossbow for communication. For the mobility commands the user applications developed in Player/Stage API are used, that run on the $\mathrm{x} 86$ computer.

\section{2) Mobility}

The mobility component of Pharos is called Proteus1 node. The proteus1 can be any choice of the various available robots, such asi Robot Create, Segway RMP50, or a customized Traxxas Stampede.

3) Localization

For the localization of nodes in Pharos testbed various devices and sensors are used like range finding sensors, digital compasses, GPS and cameras. Supporting drivers are already available in Player API for most of the sensors but for custom devices thedrivers are to be written.

\section{Scorpion: A Heterogeneous Wireless Networking Testbed}

SCORPION (Santa Cruz Mobile Radio Platform for Indoor and Outdoor Networks), is a heterogeneous wireless network testbed. It is a project initiated by the Inter-Networking Research Group (i-NRG) at UC Santa Cruz University of California [8].

\section{1) Major components}

The major components include Airlplane Node, Bus Node, Briefcase Node, and i-Robot Node as reflected in Fig. 2.

a) Airplane Node: Four autonomous airplanes and four self-stabilizing helicopters provide aerial coverage. The air crafts are controlled by Paparazzi. The air craft circles between different GPS waypoints. The nodes are mounted with 802.11 radios and can communicate with any other node in the testbed as well as can act as bridge to the disconnected region of testbed

b) Bus Node: 40 nodes are equipped with wireless radios and deployed on campus busses in order to blanket the area effectively. Nodes have mini-ITX computer running Linux, three $802.11 \mathrm{a} / \mathrm{b} / \mathrm{g}$ radios, a GPS tracking device and a 900 $\mathrm{MHz}$ radio to form network between buses and base stations deployed in campus.

c) Briefcase Node: 20 nodes are carried by students via foot or bicycle while constantly transmitting data to other 
nodes they come into contact with.

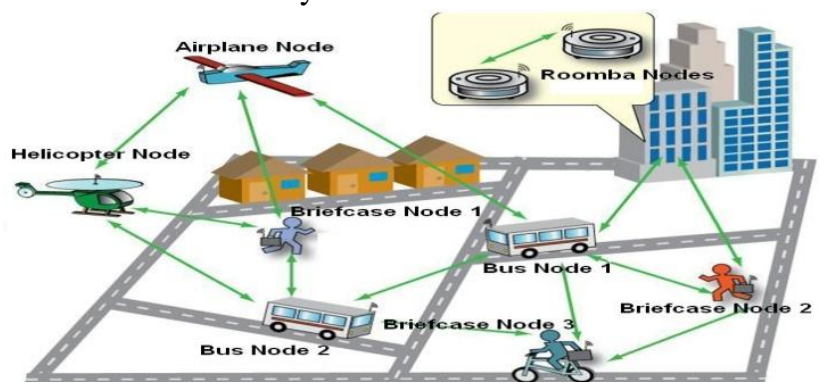

Fig. 2. Scorpion testbed with different nodes [9].

These nodes include GPS receivers, a mainboard (is a mini-ITX computer running Linux Debian Etch), and a laptop battery.

d) I-Robot Node: 20 terrestrial nodes roam the ground in an unpredictable way, making the testbed's behavior unspecified. These ground nodes are having mini-ITX computer running Linux Debian with three $802.11 \mathrm{a} / \mathrm{b} / \mathrm{g}$ radios. This hardware iscarried by iRobot Create robots with different customizations allowed.

\section{2) Mobility}

The testbed provides a heterogeneous mobility platform both in the form of on-ground robots and airborne drones. The small remote controlled autonomous aircrafts are using Paparazzi autopilot control system to stay in air and follow the flying pattern as desired. Paparazzi is free and open source project under supervision by ENAC university. The project is intended to provide highly reliable autopilot control systems [10]. The other flying nodes include small sized self-stabilizing autonomous helicopters. The on-ground robots includean autonomous iRobot Create ground robots, that are preassembled, programmable and ready to use robots. [11]. There are two non-autonomous robots, one being installed in a briefcase carried by a person in order to mimic the human mobility patterns and the other is installed in the campus bus, with GPS navigator to depict vehicle's mobility pattern.

\section{3) Localization and mobility patterns}

The Bus nodesare installed with GPS tracking, and they transmit their GPS location to various base stations throughout the campus. This information is received by the central server where the current position of bus is indicated on Google Maps. The server also publishes information on the Internet. The aforementioned setup provides a platform to test delay tolerant protocols. The briefcase nodes are carried by the people who move according to normal routines, while the nodes are continuously communicating with other nodes in the area and can be tracked using GPS. Therefore, indicating what mobility pattern a node may be following. The ground nodes can roam using random way points, changing direction randomly incase of encountering any obstacle or by following predefined points. The aerial nodes use Paparazzi autopilot system and move along different GPS waypoints, maintaining connectivity with ground nodes.

TABLE I: COMPARISON OF MOBILE WIRELESS SENSOR NETWORK TESTBEDS.

\begin{tabular}{|c|c|c|c|c|c|c|c|}
\hline & Weight & \multicolumn{6}{|c|}{ Testbeds } \\
\hline S.No & Parameters & $\boldsymbol{A}$ & $\boldsymbol{B}$ & $C$ & $D$ & $\boldsymbol{E}$ & $\boldsymbol{F}$ \\
\hline 1. & Scalability & $\mathrm{Y}$ & & & $\begin{array}{l}Y \\
Y\end{array}$ & $\mathrm{Y}$ & $\mathrm{Y}$ \\
\hline 2. & Robot based Mobility & $\mathrm{Y}$ & $\mathrm{Y}$ & - & $\mathrm{Y}$ & $\mathrm{Y}$ & $\mathrm{Y}$ \\
\hline 3. & Choreographed Mobility & - & - & $\mathrm{Y}$ & - & & \\
\hline 4. & Robots diversity & - & & - & $\mathrm{Y}$ & $\mathrm{Y}$ & $\mathrm{Y}$ \\
\hline 5. & $\begin{array}{l}\text { Auto recharging of } \\
\text { mobile nodes }\end{array}$ & $\mathrm{Y}$ & - & - & - & - & - \\
\hline 6. & Indoor deployment & & & & - & & \\
\hline 7. & Outdoor deployment & - & - & $\mathrm{Y}$ & $\mathrm{Y}$ & & \\
\hline 8. & Efficient Localization & & $\mathrm{Y}$ & - & & & \\
\hline 9. & $\begin{array}{l}\text { Remotely accessible } \\
\text { through weh }\end{array}$ & . & & - & - & - & - \\
\hline 10. & Cost Effective & & & & $\mathrm{Y}$ & & \\
\hline 11. & Repeatability & & & & - & - & - \\
\hline 12. & Run-time debugging & & - & - & - & - & - \\
\hline 13. & Real time nodes display & $\mathrm{Y}$ & & - & - & - & \\
\hline 14. & $\begin{array}{l}\text { Runtime Inter node Signal } \\
\text { quality display }\end{array}$ & $\mathrm{Y}$ & & - & - & - & - \\
\hline 15. & Node collision avoidance & $\mathrm{Y}$ & $\mathrm{Y}$ & $\mathrm{Y}$ & $\mathrm{Y}-2$ & $\mathrm{Y}$ & $\mathrm{Y}$ \\
\hline
\end{tabular}

\section{COMPARISONS}

This section contains the tabular comparison of thetestbeds included in this paperfor differentparameters. Since same feature can be provided by more than one testbed but with different service level and quality, a weight assignment is used to quantify one testbed' sprecedence over another against a specific parameter. Moreover, weights are selected 
from 1 to 3 where 1 being lowest and 3 representing highest value. Following testbeds are included in the comparison. (a) MiNT-m, (b) Mobile Emulab, (c) Ad hoc Protocol Evaluation Testbed - APE [12], (d) Pharos, (e) SCORPION, and (f) Sensei-UU [13]

Table I presents a comparison of above listed testbeds where column describe testbeds and rows indicate different parameters considered in designing of testbed. Against each parameter a certain weight is assigned in table. Blank cells indicate that certain parameter is not applicable for a testbed. Therefore, this comparisonshows that so far scorpion testbed is satisfying most parameters with more weights and hence tops the list. However, comparison of cost in scalability this testbed is expensive due to a different nature of devices and nodes used, few being quiet expensive.

Table II provides a quick summary of different types of testbeds presented in this paper.

TABLE II: A QUICKSUMMARY OF MOBILE WIRELESS SENSOR NETWORK TESTBEDS

\begin{tabular}{|c|c|c|c|c|c|}
\hline & \multicolumn{5}{|c|}{ Properties } \\
\hline Testbed & Deployment & Mobility Mechanism & Wireless \& Sensor Component & Localization Mechanism & User Interface \\
\hline A. Mint-m & Indoor & $\begin{array}{l}\text { Low cost robotic vacuum cleaner } \\
\text { called Roomba Robots. }\end{array}$ & $\begin{array}{l}\text { Router BOARD with four wireless } \\
\text { interfaces each provided by a } \\
\text { mini-PCI IEEE } 802.11 \mathrm{a} / \mathrm{b} / \mathrm{g} \text { wireless } \\
\text { card. }\end{array}$ & $\begin{array}{l}\text { Ceiling mounted } \\
\text { cameras }\end{array}$ & $\begin{array}{l}\text { MOVIE and Ns2 Nam } \\
\text { based interface }\end{array}$ \\
\hline $\begin{array}{l}\text { B.Mobile } \\
\text { Emulab }\end{array}$ & Indoor & $\begin{array}{l}\text { Acroname Garcia robots which } \\
\text { are two wheeled }\end{array}$ & $\begin{array}{l}\text { wireless } 802.11 \mathrm{~b} \text { card, the onboard } \\
\text { computer, and a Mica } 2 \text { mote }\end{array}$ & $\begin{array}{l}\text { Ceiling mounted } \\
\text { cameras and scripted } \\
\text { motion }\end{array}$ & $\begin{array}{l}\text { web-based front end } \\
\text { with option to submit } \\
\text { NS tcl script file with } \\
\text { various parameters set } \\
\text { for experiment. }\end{array}$ \\
\hline C. APE & Both & $\begin{array}{l}\text { Choreographed movement of } \\
\text { volunteers following the on-screen } \\
\text { instructions for the direction to } \\
\text { follow }\end{array}$ & $\begin{array}{l}\text { i386 computers that are preferably } \\
\text { laptops and are installed with IEEE } \\
802.11 \text { WaveLAN cards }\end{array}$ & $\begin{array}{l}\text { Depends on the position } \\
\text { of volunteers during } \\
\text { experiment }\end{array}$ & $\begin{array}{l}\text { GUI front end } \\
\text { APE-view that can } \\
\text { display topological } \\
\text { configuration of nodes } \\
\text { during the } \\
\text { experiments }\end{array}$ \\
\hline D. Pharos & Outdoor & $\begin{array}{l}\text { Can be any choice of the various } \\
\text { available robots, e-g iRobot } \\
\text { Create, Segway RMP50, or a } \\
\text { customized Traxxas Stampede }\end{array}$ & $\begin{array}{l}\text { x86 linux motherboard attached with } \\
\text { Freescale microcontroller. IEEE } \\
802.11 \text { b/g wireless card is used with } \\
5.5 \mathrm{~dB} \text { antenna. Also supports other } \\
\text { technologies like Bluetooth, } \\
\text { Crossbow for communication }\end{array}$ & $\begin{array}{l}\text { Range finding sensors, } \\
\text { digital compasses, GPS } \\
\text { and cameras. }\end{array}$ & Player/Stage \\
\hline E. SCORPION & Both & $\begin{array}{l}\text { Airplane node, Bus node, } \\
\text { Briefcase nodes, iRobot Create } \\
\text { Robots }\end{array}$ & $\begin{array}{l}\text { mini-ITX computer running LinuX } \\
\text { Debian with three } 802.11 \mathrm{a} / \mathrm{b} / \mathrm{g} \\
\text { radios. }\end{array}$ & $\begin{array}{l}\text { GPS tracking, random } \\
\text { way points, Paparazzi } \\
\text { autopilot system }\end{array}$ & $\begin{array}{l}\text { SCORPION's } \\
\text { management } \\
\text { suite }\end{array}$ \\
\hline F. Sensei-UU & Both & $\begin{array}{l}\text { Choreographed movement of } \\
\text { volunteers or on-ground } \\
\text { autonomous robots with } \\
\text { predefined motion or dynamic } \\
\text { map generation }\end{array}$ & $\begin{array}{l}\text { Sensor hosts are linux machine (e-g } \\
\text { Asus WL-500G wireless access } \\
\text { points running a small Linux } \\
\text { distribution called OpenWrt) with } \\
\text { USB ports, } 802.11 \mathrm{~b} / \mathrm{g} \text { control } \\
\text { channels }\end{array}$ & $\begin{array}{l}\text { Simultaneous } \\
\text { Localization and } \\
\text { Mapping (SLAM), } \\
\text { predefined map of } \\
\text { location, and GPS } \\
\text { points for outdoor }\end{array}$ & GUI monitor software \\
\hline
\end{tabular}

\section{CONCLUSION}

Paper presents a thorough survey of various testbeds for Mobile WSNs. Moreover, there are several ongoing efforts to develop ahighly scalable and reliable mobile WSN testbed, but still a lot of work needs to be done to meet challenges due to low fidelity radio channel and devices' mobility constraints. While mobility can be provided by robots, their cost is also a critical factor in increasing the size of the testbed. In order to make the testbedoperate 24 by 7 , some mechanisms are also required to auto-recharge the mobile nodes, without human intervention. It is also important to have a remotely accessible web based interface with the testbed so that a researcher from a remote site can connect and perform an experiment in reserved slot as usually done in ORBIT. Furthermore, current research work on submersed WSN testbeds is comparatively less than the on-ground testbeds. Moreover, most of the work on submersible testbeds is done for military applications. For example U.S navy conducted research on Autonomous Underwater Vehicles (AUV) [14]. There is a wide area of research that is yet to be conducted by sending intercommunicating robots to seabed which is not in easy human access. Medium sized aquariums can be used to deploy testbeds with robotic fish to analyze the behavior of radio communication under the water surface.

\section{REFERENCES}

[1] J. Owen. (April 2010). Free Software Tools for Robot and Sensor Applications. The Player Project at [Online]. Available: http://playerstage.sourceforge.net/.

[2] P. Mohapatra. (2004). The Quail Ridge Wireless Mesh Network (QuRiNet). Last visited in 2009. [Online]. Available: http://spirit.cs.ucdavis.edu/quailridge

[3] P. De and A. Raniwala, "MiNTm: An autonomous mobile wireless experimentation platform," in Proc International Conference on Mobile Systems, Applications and Services, Uppsala, Sweden, pp. 124-137, 2006.

[4] C. Angle, H. Greiner, and R. Brooks. (1990). iRobot. Last visited in 2011. [Online]. Available: http://www.irobot.com/us/robots/home/roomba.aspx.

[5] D. Johnson and T. S. Russ, "Mobile emulab: A robotic wireless and sensor network testbed," in Proceedings of the 25th Conference on Computer Communications(IEEE INFOCOM), vol. 2, April 2006.

[6] Acroname Robotics. (1994). Last visited in 2009. The Garcia Robots at [Online]. Available: http://www.acroname.com/garcia/garcia.html.

[7] D. Stovall, N. Paine, A. Petz, J. Enderle, C. Julien, and S. Vishwanath. "Pharos: An application-oriented testbed for heterogeneous wireless networking environments," Technical Report TR-UTEDGE-2009-006, 
The Center for Excellence in Distributed Global Environments, The University of Texas, Austin, 2009.

[8] K. Obraczka. (2004). The SCORPION Project by Inter-Netwroking Research Group (i-NRG). Last visited in 2010. [Online]. Available: http://inrg.cse.ucsc.edu/inrgwiki/Scorpion\%20Testbed.

[9] S. Bromage, C. Engstrom, J. Koshimoto, M. Bromage, S. Dabideen, M. Hu, et al., "Poster abstract: Scorpion: A heterogeneous wireless networking testbed," ACM SIGMOBILE Mob. Comput. Commun. Rev. 2009.

[10] P. Brisset. (2006). Last visited in 2010. [Online]. Available: http://paparazzi.enac.fr/wiki/Main_Page.
[11] C. Angle, H. Greiner, and R. Brooks. (1990). iRobot. Last visited on Sept 2010. [Online]. Available: http://store.irobot.com/shop/index.jsp?categoryId=3311368

[12] J. Nielsen. (November 2002). An APE Project. Uppsala University. [Online]. Available: http://apetestbed.sourceforge.net/.

[13] O. Rensfelt (September 2009). Sensei-UU: A flexible wireless sensor network testbed supporting mobile nodes. 2009. Uppsala University. [Online]. Available: http://www.it.uu.se/research/upmarc/seminars/2009-09-17-abstract.

[14] R. Hillson and C. Jones, "Evolution of an AUV Mission Simulation Testbed," in Proceedings of the 6th International Symposium on Unmanned Untethered Submersible Technology, Washington, D.C., 1989 , pp. 525-535 